breakdown products also bind to the estrogen receptor and modulate prostaglandinmediated processes. Debate continues over whether sex-hormone levels influence arthritis pathogenesis directly and whether they could be used to predict the risk of developing OA. Sowers et al., therefore, investigated the relationships between levels of estradiol (or its estrone metabolites) and risk of knee OA.

Sowers et al. measured estradiol levels in serum, and 2-hydroxyestrone and 16ahydroxyestrone levels in urine, from 842 women (mean age 42.3 years) from the Southeast Michigan Arthritis Cohort for whom radiographs of both knees were available. Women who developed knee OA had lower serum-estradiol and lower urinary 2-hydroxyestrone levels than those who did not, although, interestingly, there was only a modest correlation between estradiol and 2-hydroxyestrone levels. Women with an increased urinary 2-hydroxyestrone:16ahydroxyestrone ratio were more likely to develop incident knee OA, an association that persisted after controlling for confounding variables.

Sowers et al. speculate that high concentrations of 2-hydroxyestrone in urine could reflect inhibition of leukotriene synthesis and modulation of lipoxygenases in the arachidonic acid pathway (which is associated with pain and inflammation), rather than estrogen-receptormediated effects. They suggest that the arachidonic acid pathway could provide therapeutic targets for intervention in OA.

Original article Sowers MFR et al. (2006) Estradiol and its metabolites and their association with knee osteoarthritis. Arthritis Rheum 54: 2481-2487

\section{Antibodies to NMDA receptor 2 linked with depression in patients with SLE}

Cognitive dysfunction and depression are common manifestations of systemic lupus erythematosus (SLE), although their causes remain unclear. Receptors for $\mathrm{N}$-methyl-D-aspartate (NMDA), which bind the neurotransmitter glutamate, are known to influence mood, learning and memory; Lapteva et al., however, found no association between antibodies to NMDA receptor 2 (NR2) and cognitive dysfunction in patients with SLE, although there was a clear link between serum levels of antibodies to NR2 and depression.
Their cross-sectional, multicenter study enrolled 60 consecutive patients (mostly female and white, age range 19-67 years) with SLE, who were assessed during 1-2 sessions $\leq 2$ weeks apart. The patients were evaluated with the Beck Depression Inventory, psychiatric interview and neuroimaging studies. Patients also underwent neurocognitive assessment and were tested for the presence of several autoantibodies.

Patients with Beck Depression Inventory scores $\geq 14$ had markedly elevated titers of antibodies to NR2, even after adjustment for potentially confounding variables. This association was particularly evident in patients diagnosed with clinical depression at their psychiatric interview. The authors suggest that antibodies to NR2 contribute to depression in patients with SLE by causing overstimulation of neuronal cells, which might mimic the depressive effects of NMDA agonists. Although Lapteva et al. found no association between antibodies to NR2 and cognitive dysfunction, they speculate that long-term studies might show a dosedependent effect: low antibody titers might lead to depression, whereas high antibody titers might cause neuronal death and result in cognitive impairment.

Original article Lapteva L et al. (2006) Anti- $N$-methylD-aspartate receptor antibodies, cognitive dysfunction, and depression in systemic lupus erythematosus. Arthritis Rheum 54: $2505-2514$

\section{Multicenter cohort study of mortality in patients with SLE}

Although literature on the mortality rates of patients with systemic lupus eythematosus (SLE) is growing, it is important to consolidate and confirm previous findings. A multicenter, international, cohort study of unprecedented size has been carried out to compare the mortality rate of patients with SLE with that of the general population.

Data were collected from 9,547 patients with definite SLE, from 23 lupus centers in 7 countries, between 1958 and 2001. In 20 centers, data from patients who were deceased or lost to follow-up were matched with corresponding records on cause-of-death registers. The ratio of number of observed deaths to number of expected deaths (risk of death multiplied by matched mortality rates) was calculated. 